\title{
Stand-Alone DFIG FOC Sensitivity and Stability under Mismatched Inductances
}

\author{
I. E-Damian, J. Apsley, R. Perini, Members, IEEE, M.F. Iacchetti, G.D. Marques, Senior Members, IEEE
}

\begin{abstract}
Inaccurate machine parameters can cause orientation errors and instability in field oriented control schemes relying on model-based estimations. This paper analyzes the accuracy and stability of two field oriented control schemes for a stand-alone DFIG, where the field orientation is affected by the stator inductance and stator/mutual inductance ratio. After deriving a reduced-order model accounting for parameter mismatch, the paper deduces a formula to calculate the field orientation error as a function of the inductance mismatch, revealing parameter sensitivities. A stability analysis is then carried out proving that overestimating the stator/magnetizing inductance ratio may trigger instabilities in case of high load level, whereas underestimation allows always stable operation. The theoretical insight is supported with simulation and test results on a laboratory rig.
\end{abstract}

Index Terms - doubly fed induction generators (DFIG), fieldoriented control, stand-alone, sensitivity, stability analysis.

\section{NOMENCLATURE}

$G$ integral of the error in PI controllers

$i, v$ current, voltage

$L_{s}, L_{m}, L_{r} \quad$ stator, magnetising, rotor inductance

$R_{s}, R_{l}, R_{r} \quad$ stator, load, rotor resistance

$\gamma \quad$ orientation error

$\gamma_{s}, \gamma_{s}^{*} \quad$ actual and reference stator flux angle

$\gamma_{m}, \gamma_{s r} \quad$ rotor, slip position

$\xi_{s}, \Xi \quad$ stator inductance, inductance-ratio mismatch index

$\sigma \quad$ rotor leakage factor: $1-L_{m}{ }^{2} / L_{s} L_{r}$

$\psi_{s} \quad$ stator flux linkage

$\omega_{s}, \omega_{m}, \omega_{s r}$ stator, shaft, slip angular frequency

Subscripts and Superscripts

$d, q \quad$ stator flux oriented frame

$r, s \quad$ rotor, stator

I, $V \quad$ current, voltage loop

$\alpha, \beta \quad$ stationary frame

* reference value

- $\quad$ space vector

$\wedge \quad$ estimated quantity

\section{INTRODUCTION}

$\mathrm{D}$ OUBLY-FED induction generators (DFIGs) are a viable solution for variable-speed, constant-frequency power generation [1]. They have been widely adopted in wind energy conversion systems since the early nineties of the $20^{\text {th }}$ century because of the cheap power electronics, with a back-

I. E-Damian, J. Apsley and M.F. Iacchetti are with the University of Manchester, Manchester (UK). R. Perini is with the Department of Energy of Politecnico di Milano, Milan (IT). G. D. Marques is with the INESC-ID, Instituto Superior Técnico (IST), Universidade de Lisboa, Lisbon (PT) to-back converter rated to about $30 \%$ of the turbine power [2]. During the last twenty years a considerable amount of studies has been carried out on grid-connected DFIGs, providing full understanding on control issues and solutions. The main limitations of DFIGs during faults on the grid were recognized as soon as grid codes introduced requirements for low-voltage ride-through capability [3]. The conflict between reduced ratings for the power converter and Low-Voltage RideThrough (LVRT) capability demand has stimulated large-scale research across academia and industry, resulting in a variety of enhanced control schemes which provide adequate response during voltage sags [4]. More recently, research has focused also on operation under distorted grid voltage to assess techniques for torque ripple and stator current distortion mitigation [5]-[8]. DFIGs have also been proposed for virtual inertia implementation in wind farms, relying on the direct connection of stator to the grid and cheap power electronics [9] compared to other solutions.

DFIGs operating in stand-alone mode have been investigated too, especially for electrification in remote areas, islanded operation during black-outs, and variable-frequency onboard generation as well [10]-[23]. Stand-alone controls implement the integral of the set-point frequency as a reference angle for the stator flux linkage. Subsequently, the measured [10],[15] or estimated [13]-[14],[16]-[18] rotor position is subtracted from the flux angle to calculate the slip angle. Asymptotic field orientation along either stator flux [10]-[21] or voltage [22],[23] is then achieved in different ways. Closed-loop schemes [15]-[17] need stator flux estimation and at least one dedicated controller to force the $q$-axis flux component to zero directly. Flux estimation relies on different techniques such as stator EMF integration [14], state observers and flux-current relationships [15]-[17]. However, this adds complexity to the control layout and can potentially cause offset drift issues in the integrator or further sensitivity to parameter variation, especially when machine inductances are involved. On-line estimation of machine inductances might mitigate the impact of saturation in the main magnetic path, but at the price of an additional controller and gain tuning procedure [25]. In contrast, open-loop implementation [10] imposes the orientation just by forcing the $q$-axis rotor current to track a reference derived from the flux-current algebraic relationship. This scheme has gained wide popularity, being used also in more sophisticated control layouts including sensorless control [13], compensation for current harmonics absorbed by nonlinear loads [18]-[19], unbalance and zero-sequence currents [20] and integration with storage [21]. The same principle can be adopted in DFIG-DC systems [24].

According to [10], the open-loop FOC scheme needs one less PI controller than closed-loop controls such as [15], and avoids stator flux estimation. Both [10] and [15] however 
require the knowledge of the stator and magnetizing inductances to implement the orientation mechanism.

The effects of parameters' mismatch have been investigated mainly for grid-connected DFIGs and with major focus on the stability of the current control loops, but with little attention to the outer loops realizing field orientation in stand-alone systems. In grid-connected DFIGs, stator-flux orientation is usually achieved using the estimated stator flux angle from the integration of stator EMFs. The stator resistance is then the only parameter affecting the steady-state orientation accuracy. These schemes, however, suffer from poorly-damped and even unstable stator flux modes in the case of very high-bandwidth rotor current controllers [29],[28], regardless of parameter mismatch. In grid voltage oriented control, on the other hand, the damping of stator-flux modes is rotor-current independent [30]. The rotor current time-constant mismatch due to leakage inductance inaccuracies or rotor resistance drifts is another cause of instability which has been analyzed in [26], but such mismatches do not cause any steady-state orientation error.

Despite the major attention paid to the stability of current control loops, the impact of the inductance mismatch on the orientation mechanism in stand-alone DFIG control schemes remains substantially uninvestigated. The key peculiarity of stand-alone open-loop schemes [10], [13],[18]-[20] compared to grid-connected ones is that they set the $q$-axis reference rotor current with an algebraic constraint involving the stator current and the stator to magnetizing inductance ratio, instead of deriving it from the torque or power reference. The sensitivity study in [27] analyzes steady-state orientation inaccuracy and instability caused by mismatched inductances in MRAS observers for sensorless DFIG control, but the study focusses only on the rotor position estimation with no mention to the stator angle accuracy and stability in stand-alone mode. This paper investigates the behavior of control schemes [10] and [15] when they operate with inaccurate inductances. After building a general dynamical model, the paper derives a formula to calculate the steady-state orientation error and discusses the impact of inductances' inaccuracies on stability. The analysis proves that the stator/magnetizing inductance ratio is the main factor which affects instability. Overestimation of the inductance ratio might trigger instabilities - especially at high load levels, while an underestimation generally allows stable operation.

\section{BACKGROUND}

\section{A. DFIG equations.}

Fig. 1 shows the layout of a stand-alone DFIG system and Fig. 2 introduces the relevant reference frames for the field oriented control of the DFIG based on the stator flux linkage. The DFIG model in per unit (p.u.) is written in the stator flux oriented frame $(d q)$ as follows:

$$
\begin{gathered}
\bar{v}_{s}=R_{s} \bar{i}_{s}+\frac{1}{\omega_{b}} \frac{d \bar{\psi}_{s}}{d t}+j \omega_{s} \bar{\psi}_{s} \\
\bar{v}_{r}=R_{r} \bar{i}_{r}+\frac{1}{\omega_{b}} \frac{d \bar{\psi}_{r}}{d t}+j\left(\omega_{s}-\omega_{m}\right) \bar{\psi}_{r} \\
\bar{\psi}_{s}=L_{s} \bar{i}_{s}+L_{m} \bar{i}_{r} \quad, \quad \bar{\psi}_{r}=\frac{L_{m}}{L_{s}} \bar{\psi}_{s}+\sigma L_{r} \bar{i}_{r}
\end{gathered}
$$

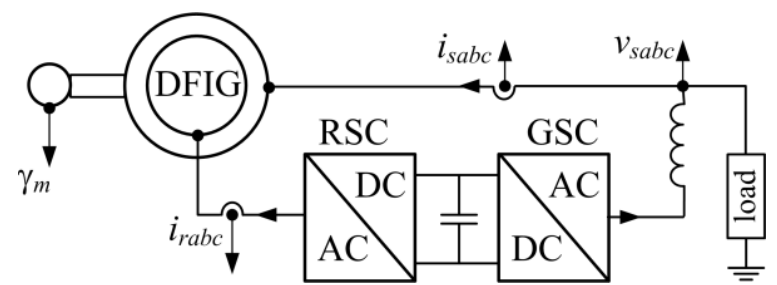

Fig. 1. Open-loop stand-alone DFIG.

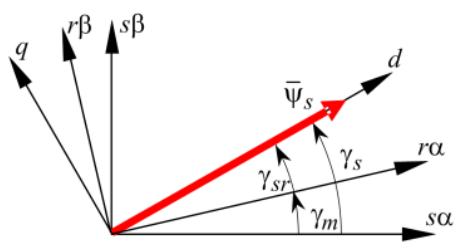

Fig. 2. Reference frames in the DFIG RSC control .

\section{B. Stand-alone FOC schemes.}

This paper considers the control schemes proposed in [10] and [15]. The general common concept for the RSC control is described in Fig. 3: the rotor position is measured by an encoder and transformed in electrical $\mathrm{rad} / \mathrm{s}$, though sensorless implementations have been proposed too in [13] and [16]. The classical inner current control based on PI regulators [2],[10],[26] is adopted to enforce the rotor current commands which come from the outer PI voltage controller and the orientation constraint, respectively.

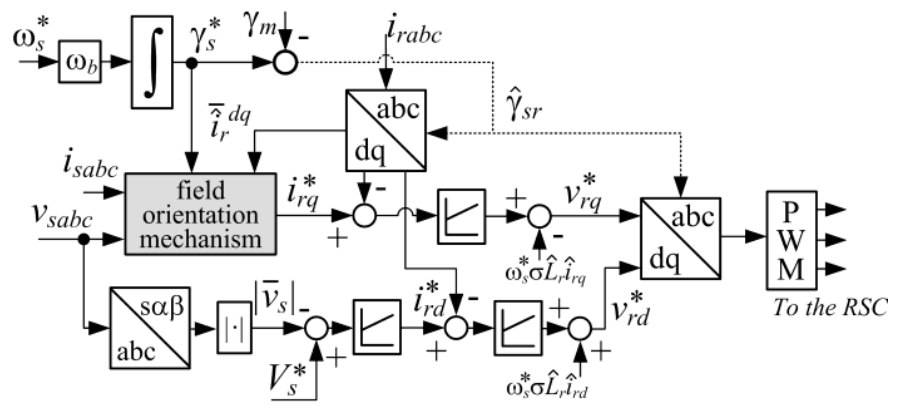

Fig. 3. Control scheme for the RSC.

Field orientation along the stator flux linkage is achieved in two steps:

1) using the integral of the reference frequency $\omega_{s}{ }^{*}$ (1 p.u. or $2 \pi \times 50 \mathrm{rad} / \mathrm{s})$ as reference angle $\gamma_{s}{ }^{*}$ for the stator frame

$$
\gamma_{s}^{*}=\omega_{b} \int \omega_{s}^{*} d t
$$

2) forcing, through the RSC, the appropriate $q$-axis reference rotor current $i_{r q}^{*}$ using a suited mechanism which depends on the considered scheme as outlined below and in Fig. 4.

Open-loop FOC [10]

In this case, $i_{r q}{ }^{*}$ is derived from (3) by assuming $\psi_{s q}=0$

$$
i_{r q}^{*[10]}=-\frac{\hat{L}_{s}}{\hat{L}_{m}} \hat{i}_{s q}=-\frac{\hat{L}_{s}}{\hat{L}_{m}} \operatorname{Im}\left(\bar{i}_{s}^{s \alpha \beta} e^{-j \gamma_{s}^{*}}\right)
$$

Closed-loop FOC [15]

Closed-loop scheme [15] adopts a Luenberger flux-observer and an extra PI controller to directly force the $q$-axis stator flux linkage to zero and generate the required $i_{r q}{ }^{*}$. 


$$
i_{r q}^{*[15]}=k_{p V}\left(0-\hat{\psi}_{s q}\right)+k_{i V} G_{\psi} \quad, \quad \frac{1}{\omega_{b}} \frac{d G_{\psi}}{d t}=0-\hat{\psi}_{s q}
$$

Eq. (7)-(8) give the flux observer formulation of [15]-[16]: $b$ (denoted with " $\sigma$ " in [16]) sets the desired dynamics. Unlike in [15], and as in [16], $\hat{R}_{s} \bar{i}_{s}$ is directly calculated with the stator current rather than via $\left(\psi_{s} / \hat{L}_{s}-\left(\hat{L}_{m} / \hat{L}_{s}\right) \bar{l}_{r}\right)$.

$$
\begin{gathered}
\frac{1}{\omega_{b}} \frac{d \overline{\hat{\psi}}_{s}}{d t}=\left(\bar{v}_{s}^{s \alpha \beta}-\hat{R}_{s} \bar{i}_{s}^{s \alpha \beta}\right) e^{-j \gamma_{s}^{*}}-b \overline{\hat{\psi}}_{s}+\left(b-j \omega_{s}^{*}\right) \overline{\hat{\psi}}_{s}^{r e f} \\
\overline{\hat{\psi}}_{s}^{r e f}=\hat{L}_{s} \bar{i}_{s}^{s \alpha \beta} e^{-j \gamma_{s}^{*}}+\hat{L}_{m} \bar{i}_{r}^{r \alpha \beta} e^{-j\left(\gamma_{s}^{*}-\gamma_{m}\right)}
\end{gathered}
$$

The caret "^" upon inductances in (4)-(7) denotes that they are generally affected by inaccuracies, due to saturation impact and limitations of commissioning procedures. The same symbol on electrical variables also reflects the inaccuracy caused by using the reference angle $\gamma_{s}{ }^{*}$ instead of the actual stator flux position $\gamma_{s}$ in reference frame transformations.

The GSC is synchronized to the local grid created by the DFIG and is in charge of regulating the power factor and the dc-link voltages. The control of the GSC is not considered in this analysis: the reader is referred to [10] for further details.

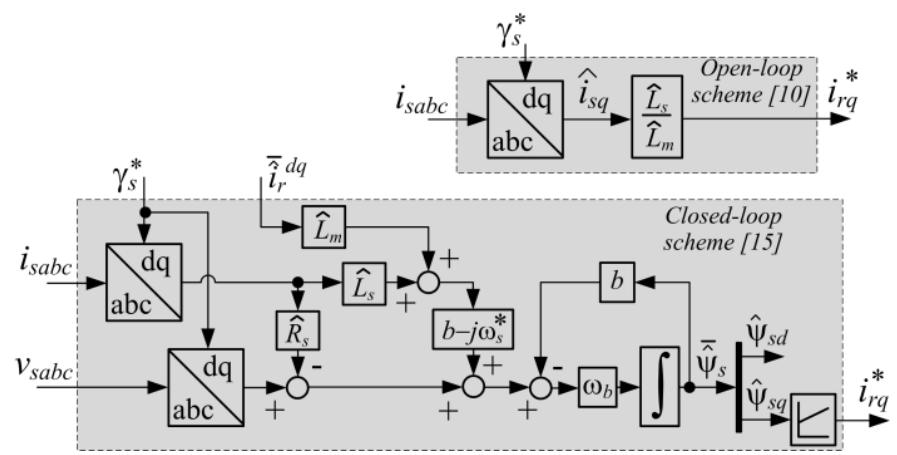

Fig. 4. Field orientation mechanisms. Top: open-loop scheme [10]. Bottom: closed-loop scheme [15], $\hat{R}_{S} \bar{i}_{S}$ is directly measured as in [16].

\section{SENSITIVITY ANALYSIS}

\section{A. Basic assumptions.}

In order to clarify the analysis approach in the easiest possible way, this paper focusses on a purely resistive standalone load. For the same reason, the GSC converter is neglected as it generally operates at unity power factor and is decoupled from the RSC by the dc-link electrolytic capacitor. Section V comments on how to extend the analysis approach to incorporate the GSC and deal with more general loads. The stator resistance is also neglected as it is normally much lower than the load resistance. Under these assumptions, the standalone DFIG is represented by the equivalent circuit in Fig. 5. Should the GSC be included in a simplified way, resistor $R_{l}$ may be the parallel of load resistor $R_{\text {load }}$ and resistor ((1$s) / s) R_{\text {load }}$ with $s=\left(\omega_{s}{ }^{*}-\omega_{m}\right) / \omega_{s}{ }^{*}=$ slip - representing the GSC according to the steady-state power balance across a DFIG. The stator voltage is established across $R_{l}$ because of the stator current flow:

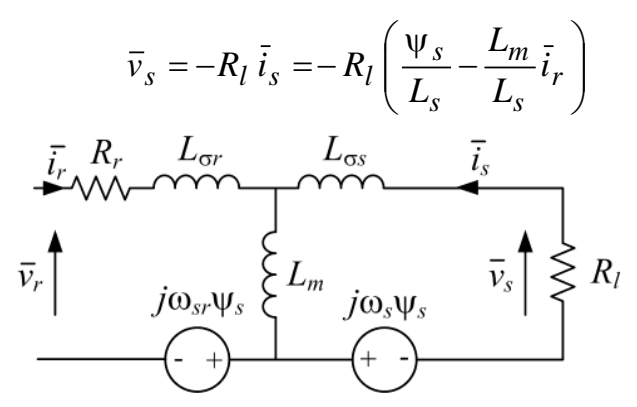

Fig. 5. Simplified equivalent circuit for the stand-alone DFIG with resistive load.

The speed is treated as a constant, as in wind-turbine-driven DFIGs the outer control loops (powers or voltage and flux) are faster than the mechanical dynamics. This assumption is usually adopted also in LVRT studies which involve large perturbations [4]. This section presents full derivations for the model of [10] whilst the details for [15] are given in the Appendix I.

\section{B. Orientation error impact on frame transformations.}

Using inaccurate inductances as well as $\gamma_{s}^{*}$ to drive the stator flux leads to an inherent orientation error in all the space-vectors referred to the $d q$ frame by the control algorithm in [10], [15]. The orientation error $\gamma$ is defined as

$$
\gamma=\gamma_{s}^{*}-\gamma_{s}
$$

where $\gamma_{s}$ is the actual stator flux angle. Orientation error $\gamma$ has a deep impact on any quantity transformed from stator or rotor frames into the control frame and vice-versa. The RSC current feed-back space vector, for instance, transforms to the fieldoriented frame as follows

$$
\overline{\hat{i}}_{r}^{d q}=\bar{i}_{r}^{s \alpha \beta} e^{-j \gamma_{s}^{*}}=\bar{i}_{r}^{d q} e^{j\left(\gamma_{s}-\gamma_{s}^{*}\right)}=\bar{i}_{r}^{d q} e^{-j \gamma}
$$

After suppressing subscripts " $d q$ " in space vectors, (11) gives

$$
\overline{\hat{i}}_{r}=\bar{i}_{r} e^{-j \gamma} \Rightarrow \begin{aligned}
& \hat{i}_{r d}=i_{r d} \cos \gamma+i_{r q} \sin \gamma \\
& \hat{i}_{r q}=-i_{r d} \sin \gamma+i_{r q} \cos \gamma
\end{aligned}
$$

Similarly, error $\gamma$ impacts on the rotor voltage applied to the DFIG via the RSC. Using an entirely similar approach to (12) and neglecting the RSC switching delay yields

$$
\bar{v}_{r}=\bar{v}_{r}^{*} e^{j \gamma} \Rightarrow \begin{aligned}
& v_{r d}=v_{r d}^{*} \cos \gamma-v_{r q}^{*} \sin \gamma \\
& v_{r q}=v_{r d}^{*} \sin \gamma+v_{r q}^{*} \cos \gamma
\end{aligned}
$$

A relationship similar to (12) holds for stator currents $\hat{\imath}_{s d}$ and $\hat{\imath}_{s q}$, so $q$-axis reference rotor current $i_{r q}{ }^{*}(5)$ is rewritten as

$$
i_{r q}^{* * 10]}=-\frac{\hat{L}_{s}}{\hat{L}_{m}} \operatorname{Im}\left(\bar{i}_{s}^{d q} e^{-j \gamma}\right)=-\frac{\hat{L}_{s}}{\hat{L}_{m}} \operatorname{Im}\left(\left(\frac{\bar{\psi}_{s}}{L_{s}}-\frac{L_{m}}{L_{s}} \bar{i}_{r}^{d q}\right) e^{-j \gamma}\right)
$$

In the last equality in (14) the stator current has been resolved in terms of stator flux and rotor current by (3-a). This is why the inner bracket in (14) comprises tuned inductances only. After introducing mismatch indices $\Xi$ and $\xi_{s}$ 


$$
\Xi=\frac{\hat{L}_{s} / \hat{L}_{m}}{L_{s} / L_{m}} \quad, \quad \xi_{s}=\hat{L}_{s} / L_{s},
$$

the $q$-axis reference rotor current (14) becomes

$$
i_{r q}^{*}[10]\left(\psi_{s}, \gamma, i_{r d}, i_{r q}\right)=\frac{\Xi}{L_{m}} \psi_{s} \sin \gamma+\Xi\left(i_{r q} \cos \gamma-i_{r d} \sin \gamma\right)
$$

Notice that $\gamma \neq 0$ causes a coupling between $q$-axis reference current and flux, $d$ - and $q$-axis actual rotor currents in (16). Equations (12), (13) and (16) are the key relationships to incorporate parameter mismatches into the dynamical model.

\section{Dynamical model.}

The most convenient formulation for the dynamical model adopts the stator-flux-oriented frame $d q$ and state variables $\left\{\psi_{s}, \gamma, G_{I d}, G_{I q}, G_{V}, i_{r d}, i_{r q}\right\}$ where " $G$ " stands for the generic error integral (either current or voltage). State equations for $\psi_{s}$ and $\gamma$ are readily derived from (1) with stator voltage (9), and with the stator current and frequency expressed as a function of $\psi_{s}, i_{r d}, i_{r q}$, and $\gamma$ with (3-a) and (4), respectively.

$$
\begin{gathered}
\frac{1}{\omega_{b}} \frac{d \psi_{s}}{d t}=-\frac{R_{l}}{L_{s}} \psi_{s}+R_{l} \frac{L_{m}}{L_{s}} i_{r d} \\
\frac{1}{\omega_{b}} \frac{d \gamma}{d t}=\omega_{s}^{*}-R_{l} \frac{L_{m}}{L_{s}} \frac{i_{r q}}{\psi_{s}}
\end{gathered}
$$

Due to $\gamma \neq 0$, (17)-(18) account for $\widehat{\Psi}_{q} \neq 0$, as caused by the parameter mismatch. The voltage PI-controller state-equation defines the integral $G_{V}$ of the stator voltage error as follows

$$
\frac{1}{\omega_{b}} \frac{d G_{V}}{d t}=V_{s}^{*}-R_{l} \sqrt{\left(\frac{\psi_{s}}{L_{s}}-\frac{L_{m}}{L_{s}} i_{r d}\right)^{2}+\left(\frac{L_{m}}{L_{s}} i_{r q}\right)^{2}}
$$

The voltage controller returns $d$-axis reference current $i_{r d}{ }^{*}$

$$
i_{r d}^{*}=k_{p V}\left(V_{s}^{*}-R_{l} \sqrt{\left(\frac{\psi_{s}}{L_{s}}-\frac{L_{m}}{L_{s}} i_{r d}\right)^{2}+\left(\frac{L_{m}}{L_{s}} i_{r q}\right)^{2}}\right)+k_{i V} G_{V}
$$

The state-equations of rotor current PI controllers define the integrals of rotor current errors built with reference currents $\left\{i_{r d}{ }^{*}, i_{r q}{ }^{*}\right\}(16)$ and (20) and feedback currents from (12)

$$
\begin{aligned}
& \frac{1}{\omega_{b}} \frac{d G_{I d}}{d t}=i_{r d}^{*}-\left(i_{r d} \cos \gamma+i_{r q} \sin \gamma\right) \\
& \frac{1}{\omega_{b}} \frac{d G_{I q}}{d t}=i_{r q}^{*}-\left(-i_{r d} \sin \gamma+i_{r q} \cos \gamma\right)
\end{aligned}
$$

The outputs of rotor current controllers added to decoupling terms $\widehat{D}_{d}$ and $\widehat{D}_{q}$ (A6) in Appendix I give the reference rotor voltages

$$
\begin{aligned}
& v_{r d}^{*}=k_{p I}\left(i_{r d}^{*}-\left(i_{r d} \cos \gamma+i_{r q} \sin \gamma\right)\right)+k_{i I} G_{I d}+\hat{D}_{d} \\
& v_{r q}^{*}=k_{p I}\left(i_{r q}^{*}-\left(-i_{r d} \sin \gamma+i_{r q} \cos \gamma\right)\right)+k_{i I} G_{I q}+\hat{D}_{q}
\end{aligned}
$$

Finally, rotor equation (2) is split into real and imaginary parts (23) rewritten in terms of $\left\{\psi_{s}, \gamma, G_{I d}, G_{I q}, G_{V}, i_{r d}, i_{r q}\right\}$ with (1) and (3). Rotor voltages $v_{r d}$ and $v_{r q}$ come from (13) with (22) for $v_{r d}{ }^{*}$ and $v_{r q}{ }^{*}$ - where $i_{r d}{ }^{*}$ and $i_{r q}{ }^{*}$ are given in (14) and (20):

$$
\begin{aligned}
& \frac{\sigma L_{r}}{\omega_{b}} \frac{d i_{r d}}{d t}=v_{r d}-\left(R_{r}+\left(\frac{L_{m}}{L_{s}}\right)^{2} R_{l}\right) i_{r d}+\omega_{s r} \sigma L_{r} i_{r q}+\frac{L_{m} R_{l}}{L_{s}^{2}} \psi_{s} \\
& \frac{\sigma L_{r}}{\omega_{b}} \frac{d i_{r q}}{d t}=v_{r q}-\left(R_{r}+\left(\frac{L_{m}}{L_{s}}\right)^{2} R_{l}\right) i_{r q}-\omega_{s r} \sigma L_{r} i_{r d}+\omega_{r} \frac{L_{m}}{L_{s}} \psi_{s}
\end{aligned}
$$

The resulting state-space model for scheme [10] comprises ODEs (17)-(18), (21) and (23). For the closed-loop scheme [15], (A4) is to be included too and governs additional state variable $G_{\psi}$ (integral of estimated flux component $\widehat{\psi}_{q}$ ). The formulation for $i_{r q}{ }^{*}$ is now (A5) instead of (16).

\section{Steady-state orientation error.}

At steady-state, all the derivatives in the state-space model are zero. Since $R_{s} \approx 0$, (17)-(19) and (1) give immediately $\omega_{s}=\omega_{s}{ }^{*}$, $\psi_{s}=V_{s}^{*} / \omega_{s}^{*}, i_{r d}=\psi_{s} / L_{m}$ and $i_{r q}=\left(L_{s} / L_{m}\right)\left(V_{s}^{*} / R_{l}\right)$. The error equation for $\gamma$ depends on the considered scheme.

Open-loop FOC [10]. Replacing (16) into (21-b) - with $d G_{I q} / d t=0$, gives

$$
\frac{\Xi}{L_{m}} \psi_{s} \sin \gamma+(\Xi-1)\left(i_{r q} \cos \gamma-i_{r d} \sin \gamma\right)=0
$$

Introducing steady state values for stator flux and rotor currents into (24) and solving with respect to $\gamma$ yields

$$
\gamma^{[10]}=\tan ^{-1}\left((1-\Xi) \frac{\omega_{s}^{*} L_{s}}{R_{l}}\right)
$$

Closed-loop FOC [15]. In this case the equation for $\gamma$ comes from (A3) after setting $d G_{\psi} / d t=0$, and is $\hat{\psi}_{q}\left(\psi_{s}, \gamma, i_{r d}, i_{r q}\right)=0$. By using the steady state values for $\psi_{s}, i_{r d}$, and $i_{r q}$, and some algebraic manipulations, $\hat{\psi}_{q}\left(\psi_{s}, \gamma, i_{r d}, i_{r q}\right)=0$ becomes

$$
\left(\frac{\omega_{s}^{*}}{b} \frac{\Xi-\xi_{s}}{\Xi}+\xi_{s} \frac{1-\Xi}{\Xi} \frac{\omega_{s}^{*} L_{s}}{R_{l}}\right) \cos \gamma-\xi_{s}\left(\frac{1}{\Xi}+\frac{\omega_{s}^{*}}{b} \frac{1-\Xi}{\Xi} \frac{\omega_{s}^{*} L_{s}}{R_{l}}\right) \sin \gamma=0
$$

Solving (26) with respect to $\gamma$ yields

$$
\gamma^{[15]}=\tan ^{-1}\left(\frac{\frac{\left.\omega_{s}^{*} \frac{1}{b} \frac{\xi_{s}}{\left(\Xi-\xi_{s}\right.}\right)+(1-\Xi) \frac{\omega_{s}^{*} L_{s}}{R_{l}}}{1+\frac{\omega_{s}^{*}}{b}(1-\Xi) \frac{\omega_{s}^{*} L_{s}}{R_{l}}}}{)}\right)
$$

As expected, (25) and (27) predict $\gamma=0$ in case of no mismatch, namely when $\Xi=1$ and $\xi_{s}=1$. 
Steady-state error integrals $G_{I d}, G_{I q}$ and $G_{V}$ come from (22) and (20) respectively, where reference voltages are found by inverting (13) with $\left\{v_{r d}{ }^{*}, v_{r q}{ }^{*}\right\}$ from (23) and replacing steadystate $\psi_{s}, i_{r d}, i_{r q}$ and $\gamma$ from (25) or (27), being at steady-state $\bar{\imath}_{r}^{*}=\overline{\hat{t}}_{r}$ (12). The steady-state value of $G_{\psi}$ - only relevant to scheme [15], is found from (A5).

Equations (16) and (25) show that the scheme in [10] is only sensitive to $\Xi$, i.e. to the accuracy of the inductance ratio $\hat{L}_{s} / \hat{L}_{m}$. In contrast, and according to (A3) and (27), the closedloop scheme [15] is sensitive to both $\Xi$, and $\xi_{s}$ namely to $\hat{L}_{s}$ and $\hat{L}_{m}$ individually. The cut-off frequency $b$ of the flux Luenberger observer, however, has an important impact on the sensitivity to $\xi_{s}$ (i.e. $\hat{L}_{s}$ ). When $b>>\omega_{s}^{*}(=1$ p.u.) the effect of $\xi_{s}$ becomes less and less significant and (27) tends to (25).

\section{STABILITY ANALYSIS}

Stability is assessed with the eigenvalue analysis on the state matrix associated with equations (17)-(18), (21), (23), and, for [15], also (A4), once they are linearized around a steady-state point derived with the procedure in Section III-D.

It is worth mentioning that a further reduction of the model in Section III-C is possible by assuming rotor current controllers with infinite dynamics and then reducing the set of statevariables to $\left\{\psi_{s}, \gamma, G_{V}\right\}$, plus $G_{\psi}$ for [15]. This simplified approach allows stability conditions to be worked out analytically and shows that instability in the open-loop scheme [10] occurs if and only if $\Xi>1$, regardless to load level, machine parameters and voltage controller design. Although the stability condition $\Xi \leq 1$ is too restrictive from a quantitative point of view, it outlines the scenario $\Xi>1$ as potentially critical. In the reality, the finite dynamics of rotor current controllers enlarges the stability domain towards $\Xi$ values slightly higher than unity. The beneficial impact of finite current bandwidth is also well-known for the stator-flux stability [28] in grid-connected DFIGs. This is why current controllers have to be taken into account and their bandwidth is considered as a main input for the stability analysis, along with mismatch index $\Xi$ and p.u. load resistance $R_{l}$.

Open-loop FOC [10]. Fig. 6 shows the stability regions (shaded areas) of the scheme [10] traced in the $R_{l}-\Xi$ plane for different current controller bandwidths, considering a $3 \mathrm{MW}$ DFIG (top plot) and a $15 \mathrm{~kW}$ DFIG (bottom plot) at synchronism. Machine parameters are given in the Appendix. The stability region boundaries are obtained by detecting points $\left(R_{l}, \Xi\right)$ where the critical eigenvalue of the linearized model crosses the imaginary axis and moves into the righthand region of the complex plane.

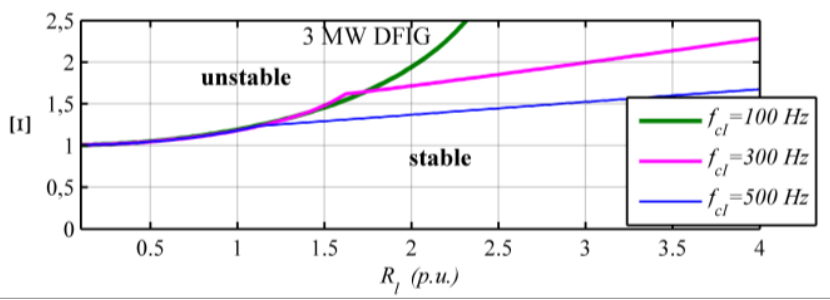

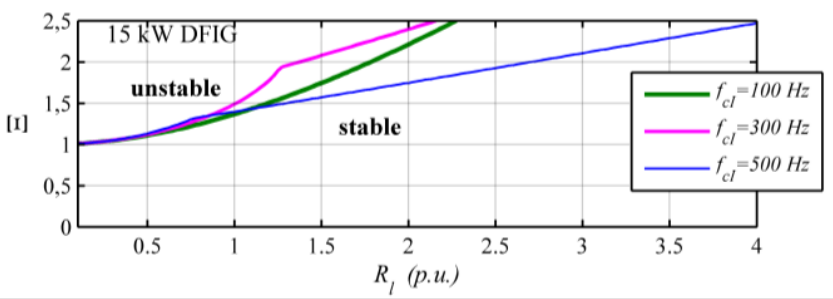

Fig. 6. Stability charts for open-loop FOC scheme [10] at synchronism. Top: 3MW machine; bottom: $15 \mathrm{~kW}$ machine.

Closed-loop FOC [15]. Although due to (19) this scheme is sensitive to both $\Xi$ and $\xi_{s}$, index $\xi_{s}$ has not significant impact on the stability as long as $\Xi=1$. For this reason, stability charts are presented in Fig. 7 assuming $\xi_{s}=1$, and a standard design of the flux observer with $b=2$ p.u. [15]-[16].

The extremely large and unrealistic $\Xi$-range explored in Figs. 6 and 7 has been deliberately chosen to show the effect of the current controller bandwidth. From a practical point of view, the most interesting outcome from Figs. 6 and 7 is that the system can become unstable only when $\Xi>1$, i.e. when the inductance ratio is overestimated. It can be concluded that schemes [10] and [15] perform in a similar way when operated close to rated-load conditions. Also, large DFIGs are more prone to instability, especially operating at full load or slightly overloaded: in that condition stability boundaries coalesce into a unique curve which tends to $\Xi=1$ as $R_{l} \rightarrow 0$. Underestimating the inductance ratio, on the other hand, does not cause instability, except for the pointless scenario $\Xi=0$.
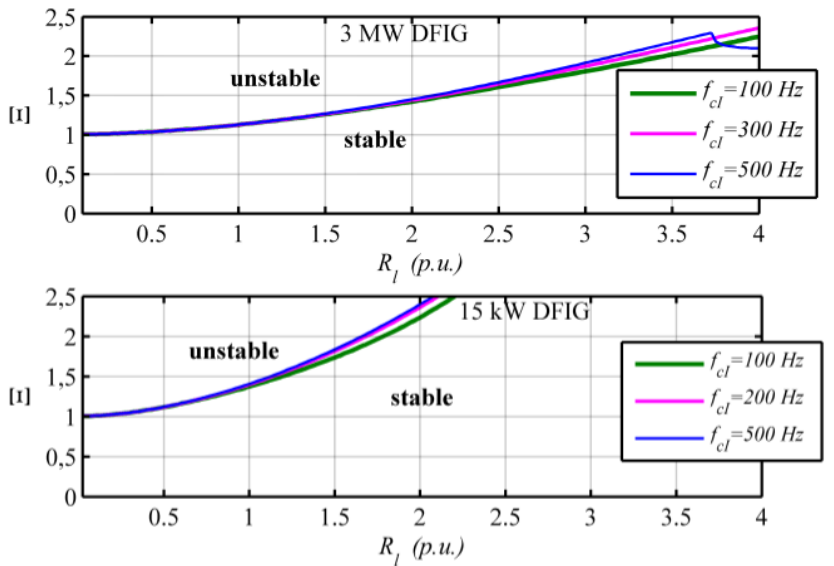

Fig. 7. Stability charts for closed-loop scheme [15] at synchronism. Top: 3MW machine; bottom: $15 \mathrm{~kW}$ machine.

Stability regions are slightly affected by speed in different ways depending on the control scheme. In the open-loop scheme [10] they slightly expand and contract at sub- and super-synchronous speed, respectively. The closed-loop scheme in [15] exhibits an opposite behavior. Large machines working at full load and at the bounds of the speed range may become unstable for values $\Xi$ closer to unity.

\section{EXTENSION TO GENERAL LOADS}

The conclusions in Sections III-IV were derived under the assumption of purely resistive load and GSC with fast dynamics and working at unity power factor. In the reality, the stand-alone system is likely to supply generic resistive 
inductive loads and the GSC may or may not compensate part of the reactive power. The complete analysis of this case requires a much higher order model than that in Section III, in order to describe the load and the GSC filter and control dynamics. The load may be described with its operational (dynamic) impedance, though the changes in frequency - due to the dynamics of $\gamma$ as in (18), produce $\Delta \omega$-dependent terms in the linearization. Despite being feasible, this approach prevents general conclusions on the stability to be drawn, because the eigenvalue analysis is to be applied to a specific configuration with many parameters. Nonetheless, the steadystate orientation error can be still derived for a generic passive load in a relatively easy way, as at steady-state the "load + GSC" system is simply described by its equivalent impedance seen from stator terminals. It is more convenient to retain the parallel load resistor $R_{l}$ and adopt power factor $P F_{s}$ at the stator terminals as an extra degree of freedom fully describing the combined effect of an inductive load and a specific setpoint for the reactive power shared by the GSC. Equation (25) and (27) can be generalized as in (28) by starting from (A3) and using the procedure explained in Section III-D with the updated steady-state d-axis current $i_{r d}=\psi_{s} / L_{m}+$ $\left(L_{s} / L_{m}\right)\left(V_{s}^{*} / R_{l}\right)\left(R_{l} / X_{l||}\right)$ with $R_{l} / X_{l||}=\left(1-P F_{s}^{2}\right)^{1 / 2} / P F_{s}$ - the other relevant steady-state values being identical to those given at the beginning of Section III-D. Quantity $X_{l||}$ is a reactance in parallel with $R_{l}$ and representing the reactive component of the load: for a purely resistive load, $P F_{s}=1$ and $X_{l||}=\rightarrow \infty$.

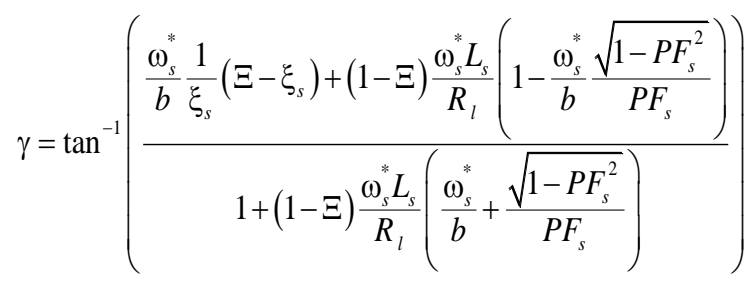

If $P F_{s}=1$, (28) reduces to (27), and then to (25) if $b / \omega_{s}{ }^{*} \rightarrow \infty$.

Fig. 8 compares the steady-state orientation errors versus $P F_{s}$ for scheme [10] and [15] and for the 3MW and 15MW WRIMs respectively, according with (28). In scheme [10], leading (negative) $P F_{s}$ and $\Xi<1$ or lagging (positive) $P F_{s}$ and $\Xi>1$ have a strong and symmetrical impact on the trend of orientation error $\gamma$. This symmetry is not retained in [15], unless $b>>\omega_{s}{ }^{*}$.
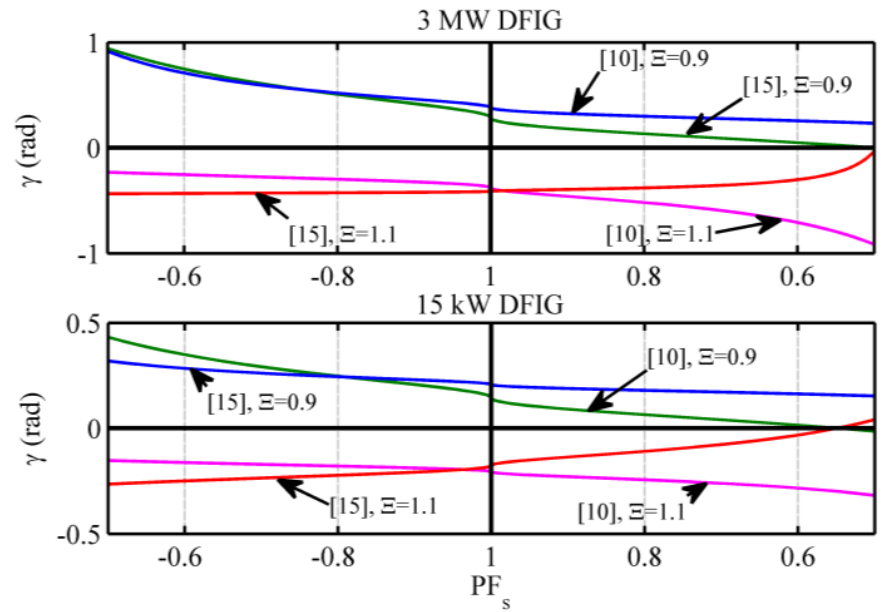

Fig. 8. Impact of the load power factor on the steady-state orientation error of schemes [10] and [15].

\section{SimUlation STUdIES}

Simulations are carried out with a state-space MatlabSimulink model using the two machines of different ratings presented in the Appendix to show the impact on stability. The $15 \mathrm{~kW}$ machine is also used in the test-rig. Frequency and voltage set-points are set to 1 p.u., current and voltage PI controllers are designed with a bandwidth of $200 \mathrm{~Hz}$ and 10 $\mathrm{Hz}$ respectively. Simulations explore the impact of different mismatch index values $\Xi$ at full load and synchronism. Stability is assessed by analyzing orientation error $\gamma$ and stator voltage magnitude $v_{s}$ responses under step changes of the inductance ratio mismatch index $\Xi$. In order to prove that $\Xi<1$ does not trigger any instability, a double step from $\Xi=1$ to $\Xi=0.8$ and then up to $\Xi=1.05$ (or $\Xi=1.27$ for the $15 \mathrm{~kW} \mathrm{DFIG)}$ is delivered first, showing fairly damped responses. $\Xi$ is subsequently increased with small steps until oscillations occur at some value $\Xi>1$. Figs. 8 to 10 present simulation results.

Fig. 9 shows the behavior of the open-loop scheme [10] at full load ( $R_{l}=1$ p.u.) under step changes in $\Xi$ for a $3 \mathrm{MW}$ (top) and $15 \mathrm{~kW}$ machine (bottom). Markers "o" depict analytical values of $\gamma$ from (25), showing an excellent agreement with simulated values. Instability is triggered at $\Xi=1.14$ and $\Xi=1.26$ respectively and causes growing oscillations in $\gamma$ and $v_{s}$.

Fig. 10 shows the behavior of the closed-loop scheme [15] under the same conditions used for Fig. 9 The instability is triggered for $\Xi=1.11$ (3MW DFIG) and $\Xi=1.26$ (15kW DFIG) respectively and gives rise to growing oscillations. The stability limits predicted by the eigenvalue analysis are $\Xi=1.113$ and $\Xi=1.265$ respectively. The simulated orientation error values and those calculated with (27) show little discrepancies for the $15 \mathrm{~kW}$ machine: they are likely to be due to the impact of stator resistance on the flux observer (7)-(8).

Finally, Fig. 11 presents the response of the closed-loop scheme [15] under sudden changes of $\xi_{s}$ from 1 to 0.6 and then to 1.4 , with $\Xi=1$ and for the 3MW DFIG. No instability and no oscillations appear in the response.

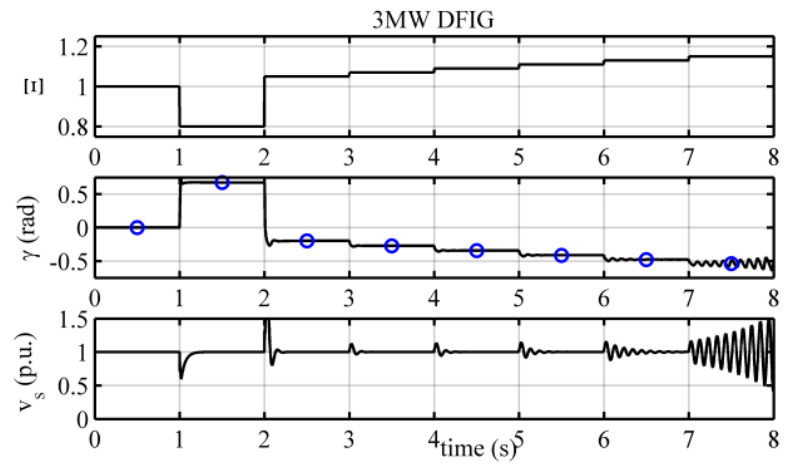



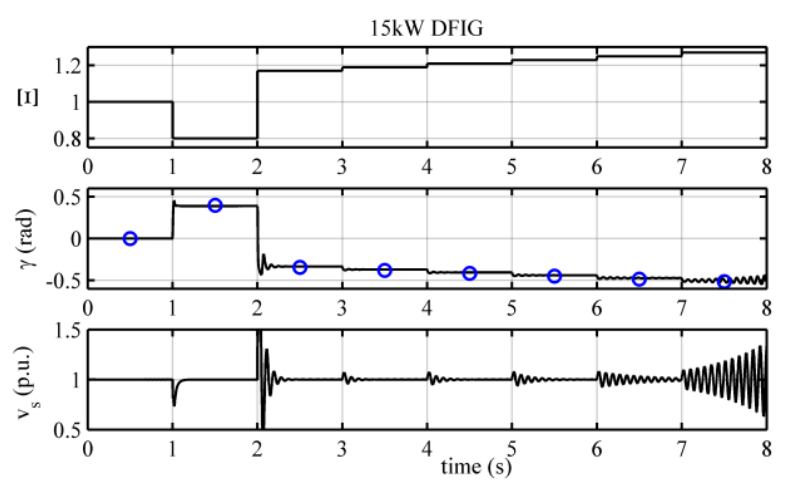

Fig. 9. Response of open-loop FOC scheme [10] to step changes in $\Xi$. Top: 5MW machine; bottom: $15 \mathrm{~kW}$ machine.
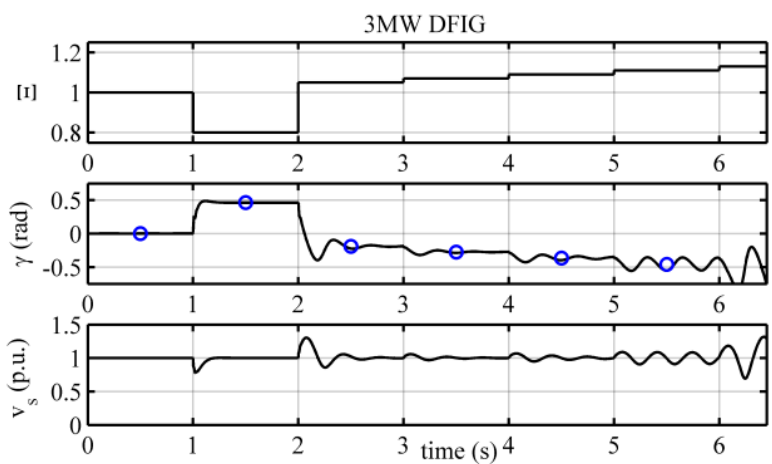

$15 \mathrm{~kW}$ DFIG
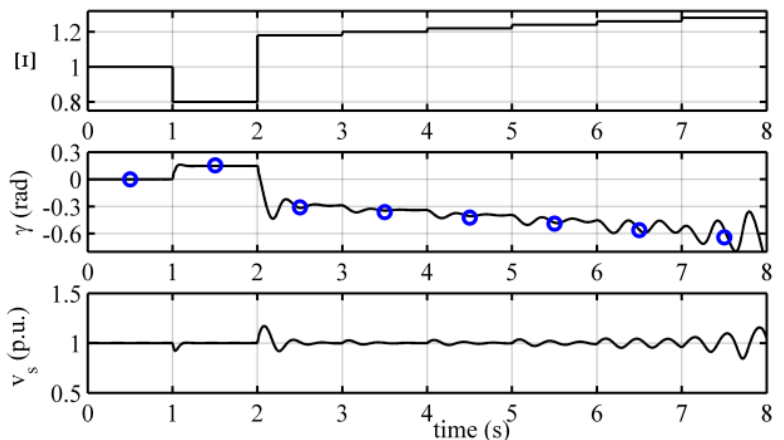

Fig. 10. Response of closed-loop FOC scheme [15] to step changes in $\Xi$. Top: 3MW machine; bottom: $15 \mathrm{~kW}$ machine.
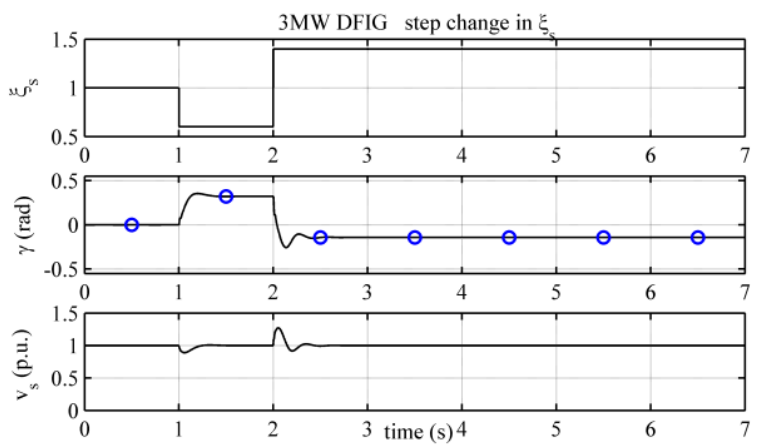

Fig. 11. Response of closed-loop scheme [15] to step changes in $\xi_{\text {s. }}$ (3MW DFIG).

\section{EXPERIMENTAL RESULTS}

The experimental setup comprises a $15 \mathrm{~kW}$ wound-rotor induction machine driven by a DC motor drive and connected to a resistor bank acting as an ac load. The RSC is controlled with the DSpace DS1103 platform with a switching frequency of $5 \mathrm{kHz}$. The dc-link is energized using a diode-bridge supplied from the $400 \mathrm{~V}$ mains and protected with a braking chopper to handle the energy flow coming from the rotor in super-synchronous operation. Current and voltage measurements are filtered with $1^{\text {st }}$-order low-pass filters with cut-off frequency set at $700 \mathrm{~Hz}$. The bandwidths of current and voltage controllers are set to $200 \mathrm{~Hz}$ and $10 \mathrm{~Hz}$ respectively. The stator load is a bank of five $58 \Omega$ resistors in parallel per-phase, each with rated current of $2 \mathrm{~A}$ ( 0.1 p.u.), bringing the overall load resistance down to 1 p.u. In order not to exceed current limits in the resistors, the reference voltage is set at 0.5 p.u..

Fig. 12 shows the experimental response of the orientation error and stator voltage magnitude with the open-loop scheme [10] when $\Xi$ is varied in steps (by adjusting the ratio $\hat{L}_{s} / \hat{L}_{m}$ in the control algorithm), first down to 0.7 , then up to 1.2 and finally with increments of 0.02 . The instability occurs at $t \approx 37$ $\mathrm{s}$ (self-excited oscillations), and the VSI trips at $t \approx 47 \mathrm{~s}$. The second subplot in Fig. 12 compares the experimental trend of $\gamma$ with theoretical values from (25) - marked with " $\mathbf{x}$ ", and show a very good agreement. The orientation error causes $\hat{\imath}_{r d}$ and $\hat{\imath}_{r q}$ estimated by the control to change, though the correct rotor currents $i_{r d}$ and $i_{r q}$ obviously remain constant because stator voltage and load are unvaried. Fig. 12 also confirms that values of $\Xi$ less than unity are not harmful. The experimental instability limit for $\Xi$ is 1.28 , slightly higher than the theoretical prediction of 1.26 .
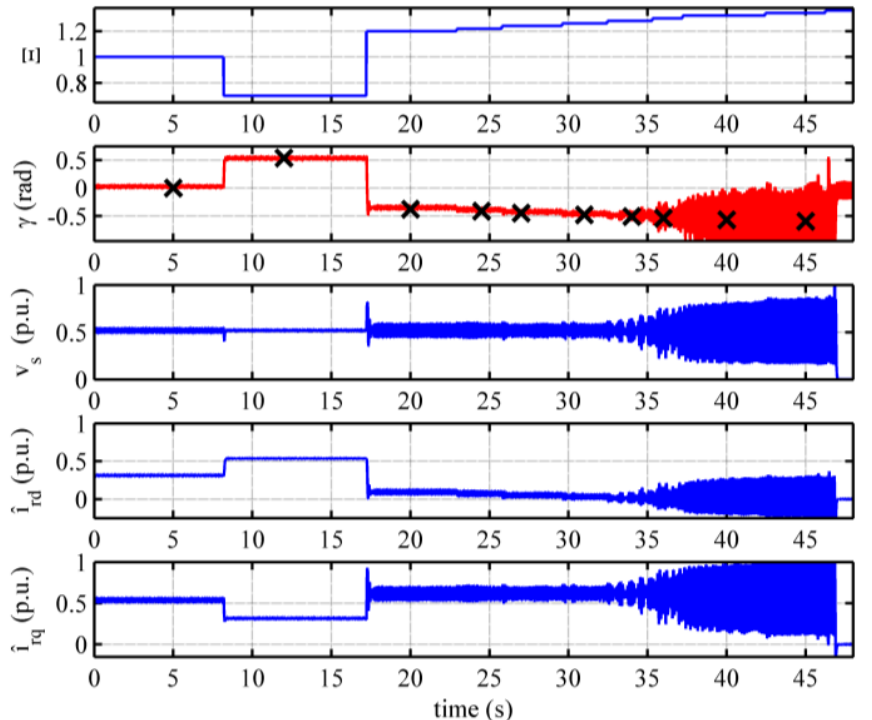

Fig. 12. Experimental response of open-loop FOC scheme [10] to step changes in $\Xi$ with the $15 \mathrm{~kW}$ machine. Speed $=1.1$ p.u.

Fig. 13 shows the experimental response of the closed-loop scheme [15] after step changes of $\Xi$ similar to those in Fig. 12. 

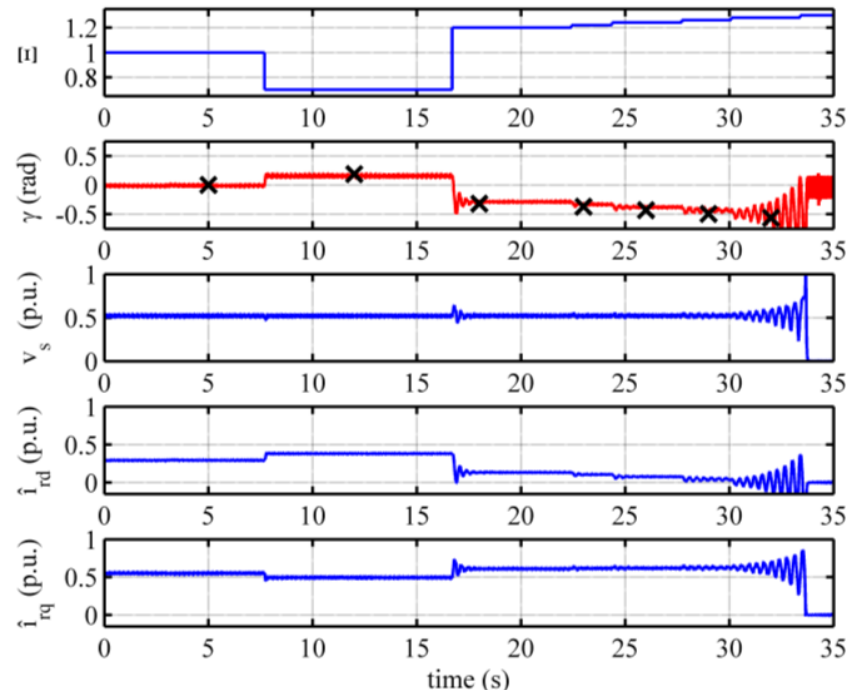

Fig. 13. Experimental response of closed-loop FOC scheme [15] to step changes in $\Xi$ with the $15 \mathrm{~kW}$ machine. Speed $=0.83$ p.u..

Here, the instability occurs at $t \approx 30 \mathrm{~s}$ and the inverter trips at $t \approx 34 \mathrm{~s}$. The experimental instability limit for $\Xi$ is now 1.28 , yet again close to the theoretical prediction of 1.27 (Fig. 7bottom). The second subplot in Fig. 13 compares the experimental trend of orientation error $\gamma$ with theoretical values form (27) (markers $\mathbf{x}$ ): the analytical prediction is fairly accurate though for $\Xi>1$ it tends to overestimate the error (e.g. for the last stable value $\Xi=1.26: \gamma_{e x p}=0.44 \mathrm{rad}, \gamma_{t h}=0.49 \mathrm{rad}$ ). This behavior has been observed also in simulation results (see Fig. 10-bottom), and the frequency of the unstable oscillations is comparable, considered the different scale for the time axes.

The impact of a mismatch in $\xi_{s}$ on the closed-loop scheme [15] is investigated in Fig. 14, which shows the experimental response to large step changes of $\xi_{s}$ from 1 to 0.7 and then from 1 to 1.5 and vice-versa, while $\Xi$ is kept at unity. These perturbations result in large variations of error $\gamma$ closely predicted by (27) - as shown by markers $\mathbf{x}$, but no instability occurs. Further tests were carried out at different speeds confirming the theory outlined in Sections III and IV.
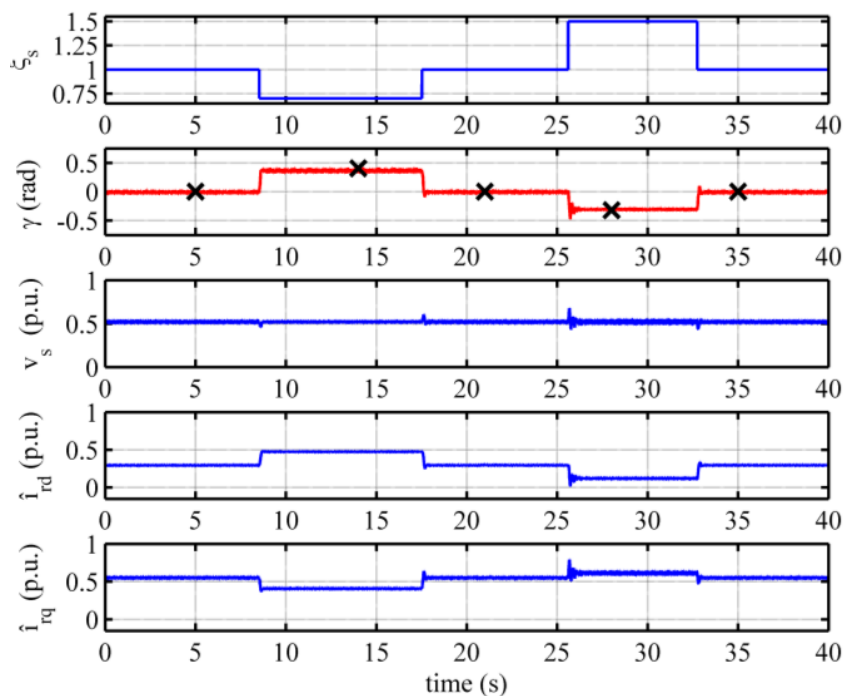

Fig. 14. Experimental response of closed-loop FOC scheme [15] to step changes in $\xi_{s}$ with the $15 \mathrm{~kW}$ machine. Speed $=0.83$ p.u.

\section{CONCLUSIONS}

A study of how parameter mismatches affect accuracy and stability of two FOC schemes for stand-alone DFIGs was presented in this paper. A reduced-order dynamical model accounting for parameter mismatch was derived leading to a formula for predicting the steady-state orientation error and showing that the accuracy and stability are mainly affected the mismatch in the stator/rotor inductance ratio, and by load resistance. The model was validated by simulations and experiments, proving that an overestimation of the inductance ratio may trigger instabilities, especially at high load levels. This might suggest implementing an inductance ratio slightly reduced with respect to the rated value, leading to a small inaccuracy in the field orientation but providing some margin against instability. The proposed analysis framework has been validated by experiments on a $15 \mathrm{~kW}$ test-rig and can be easily extended to other stand-alone DFIG controls simply by modifying the equation for the field orientation mechanism.

\section{APPENDIX I}

In order to reduce the model order, the flux observer is assumed to have infinite bandwidth: this assumption is consistent with the design guidelines in [15]-[16], as the flux observer is to be designed with a bandwidth much higher than that of the current loops. Therefore, (7) is to be rewritten at steady-state with $\hat{R}_{s}=0$ and $\bar{v}_{s}^{s \alpha \beta}=j \omega_{s}^{*} \psi_{s}$, and using (8).

$j \omega_{s}^{*} \psi_{s} e^{-j \gamma_{s}^{*}}-b \overline{\hat{\psi}}_{s}+\left(b-j \omega_{s}^{*}\right)\left(\hat{L}_{s} \bar{i}_{s}^{s \alpha \beta}+\hat{L}_{m} \bar{i}_{r}^{r \alpha \beta} e^{-j \gamma_{m}}\right) e^{-j \gamma_{s}^{*}}=0$

Treating (A1) with the same approach used in (11) returns

$$
\begin{aligned}
& b \overline{\hat{\psi}}_{s}=\left(j \omega_{s}^{*} \psi_{s}+\left(b-j \omega_{s}^{*}\right)\left(\hat{L}_{s} \bar{i}_{s}^{d q}+\hat{L}_{m} \bar{i}_{r}^{d q}\right)\right) e^{-j \gamma} \\
& =\left(j \omega_{s}^{*} \psi_{s}+\left(b-j \omega_{s}^{*}\right)\left(\hat{L}_{s}\left(\frac{\psi_{s}}{L_{s}}-\frac{L_{m}}{L_{s}} \bar{i}_{r}^{d q}\right)+\hat{L}_{m} \bar{i}_{r}^{d q}\right)\right) e^{-j \gamma}
\end{aligned}
$$

The imaginary part of (A2) rewritten with the indexes (15) is

$$
\begin{array}{r}
\hat{\psi}_{r q}\left(\psi_{s}, \gamma, i_{r d}, i_{r q}\right)=\xi_{s}\left(\psi_{s}+\left(i_{r d}+\frac{\omega_{s}^{*}}{b} i_{r q}\right) L_{m} \frac{1-\Xi}{\Xi}\right) \sin \gamma+ \\
+\left(\left(1-\xi_{s}\right) \frac{\omega_{s}^{*}}{b} \psi_{s}+\left(i_{r q}-\frac{\omega_{s}^{*}}{b} i_{r d}\right) L_{m} \xi_{s} \frac{1-\Xi}{\Xi}\right) \cos \gamma
\end{array}
$$

The model for [15] includes an extra state equation similar to (19) for the PI controller forcing $q$-axis flux (A3) to zero:

$$
\frac{1}{\omega_{b}} \frac{d G_{\psi}}{d t}=-\hat{\psi}_{r q}\left(\psi_{s}, \gamma, i_{r d}, i_{r q}\right)
$$

Finally, $i_{r q}{ }^{*}$ in [15] is simply the output of the flux controller

$$
i_{r q}^{*[15]}=k_{p V} \hat{\psi}_{r q}\left(\psi_{s}, \gamma, i_{r d}, i_{r q}\right)+k_{i V} G_{\psi} \quad \text { closed loop [15] }
$$

Decoupling terms $\widehat{D}_{d}$ and $\widehat{D}_{q}$ in (22) for both [10] and [15] are 


$$
\begin{aligned}
& \hat{D}_{d}=\left(\omega_{s}^{*}-\omega_{m}\right) \sigma L_{r}\left(-i_{r d} \sin \gamma+i_{r q} \cos \gamma\right) \\
& \hat{D}_{q}=-\left(\omega_{s}^{*}-\omega_{m}\right) \sigma L_{r}\left(i_{r d} \cos \gamma+i_{r q} \sin \gamma\right)
\end{aligned}
$$

\section{APPENDIX II}

3MW DFIG parameters: 690V, 3050A, $50 \mathrm{~Hz}$, 4-pole, $L_{m}=3.8$ p.u., $L_{r}=4$ p.u., $L_{s}=4$ p.u., $R_{r}=0.007$ p.u., $R_{S}=0.007$ p.u..

$15 \mathrm{~kW}$ DFIG data (test-rig): $400 \mathrm{~V}, 20 \mathrm{~A}, 50 \mathrm{~Hz}, 6$ poles, turns ratio $0.97, L_{m}=2.00$ p.u., $L_{r}=2.10$ p.u., $L_{s}=2.10$ p.u., $R_{r}=0.050$ p.u., $R_{s}=0.028$ p.u..

\section{REFERENCES}

[1] R. Cárdenas, R. Peña, S. Alepuz, G. Asher, "Overview of control systems for the operation of DFIGs in wind energy applications," IEEE Trans. Ind. Electron., vol. 60, n 7, pp. 2776-2798, July 2013.

[2] R. Pena, J. C. Clare, and G. M. Asher, "Doubly fed induction generator using back-to-back PWM converters and its application to variablespeed wind-energy generation," Proc. Inst. Elect. Eng. -Elect. Power Appl., vol. 143, pp. 231-241, May 1996.

[3] Eltra, Specifications for Connecting Wind Farms to the Transmission Network, ELT 1999-411a, 2004.[Online].Available: http://www.eltra.dk.

[4] V. F. Mendes, C. Venicio de Sousa, S. Rocha Silva, B. C. Rabelo, W. Hofmann, "Modelling and ride-through control of doubly fed induction generators during symmetrical voltage sags," IEEE Trans. En. Conv., vol. 26, No. 4, Dec. 2011 pp. 1161 - 1171.

[5] L. Fan, S. Yuvarajan, and R. Kavasseri, "Harmonics analysis of a DFIG for a wind energy conversion system," IEEE Trans. Energy Convers., vol. 25, no. 1, pp. 181-190, Mar. 2010.

[6] J. Hu, H. Nian, H. Xu, and Y. He, "Dynamic modeling and improved control of DFIG under distorted grid voltage conditions," IEEE Trans.Energy Convers., vol. 26, no. 1, pp. 163-175, Mar. 2011.

[7] Changjin Liu, F. Blaabjerg, Wenjie Chen, Dehong Xu, "Stator current harmonic control with resonant controller for doubly fed induction generator," IEEE Trans. Power Electron, vol. 27, no. 7, pp. 3207-3220, July 2012.

[8] H. Xu, J. Hu, and Y. He, "Operation of wind-turbine-driven DFIG systems under distorted grid voltage conditions: Analysis and experimental validations," IEEE Trans. Power Electron., vol. 27, no. 5, pp. 2354-2366, May 2012.

[9] M. F. M. Arani, E. F. El-Saadany, "Implementing Virtual Inertia in DFIG-Based Wind Power Generation," IEEE Trans. Pow. Systems, vol. 28, no. 2, pp. 1373-1384, May 2013.

[10] R. Pena, J. C. Clare, and G. M. Asher, "A doubly fed induction generator using back-to-back PWM converters supplying an isolated load from a variable speed wind turbine," Proc. Inst. Elect. Eng.-Elect. Power Appl., vol. 143, no. 5, pp. 380-387, Sep. 1996.

[11] G. Iwansky, W. Koczara, "DFIG-based power generation system with UPS function for variable-speed applications," IEEE Trans. Ind. Electron., vol. 55, no. 8, pp. 3047-3054, Aug. 2008.

[12] T. Feehally, J. M. Apsley, "The douby fed induction machine as an areo generator," IEEE Trans. Ind. Appl., vol. 51, no. 4, pp.3462-3471, MayJun. 2015.

[13] R. Cárdenas, R. Pena, J. Proboste, G. Asher, J. Clare, "MRAS Observer for Sensorless Control of Standalone Doubly Fed Induction Generators," IEEE Trans. on En. Conv., vol. 20, No. 4, Dec. 2005 pp. 710 - 717.

[14] M. Pattnaik, D. Katsha, "Harmonic compensation with zero-sequence load voltage control in a speed-sensorless DFIG-based stand-alone VSCF generating system," IEEE Trans. Ind. Electron., vol. 60, no. 12, pp. 5506-5514, Dec. 2013.

[15] D. Forchetti, J. A. Solsona, G. O. Garcia, and M. I. Valla, "A control strategy for stand-alone wound rotor induction machine," Elect. Pow. Syst. Res., vol. 77, no. 2, pp. 163-169, Feb. 2007.

[16] D. Forchetti, G. Garcia, M. I. Valla, "Adaptive Observer for Sensorless Control of Stand-Alone Doubly Fed Induction Generator," IEEE Trans. on Ind. Electronics, Vol. 56, № 10, pp. 4174 - 4180, Oct. 2009.

[17] M. Szypulsky, G. Iwansky, "Sensorless state control of stand-alone doubly fed induction generator supplying nonlinear and unbalanced loads," IEEE Trans. En. Conv., vol. 31, no. 4, Dec. 2016, pp. 1530-1538.

[18] A. Kumar Jain, V.T. Ranganathan, "Wound Rotor Induction Generator with Sensorless Control and Integrate Active Filter for Feeding
Nonlinear Loads in a Stand-Alone Grid," IEEE Trans. on Ind. Electron., vol. 55, No.1, Jan. 2008 pp. $218-228$.

[19] Van-Tung Phan, Hong-Hee Lee, "Control strategy for harmonic elimination in stand-alone DFIG applications with nonlinear loads," IEEE Trans. Pow. Electron, vol. 26, no. 9, Sept. 2011, pp. 2662-2675.

[20] G. Carrasco, C. A. Silva, R. Pena, R. Cardenas, " Control of a four-leg converter for the operation of a DFIG feeding stand-alone unbalanced loads," IEEE Trans. Ind. Elect., vol. 62, No.7, Jul. 2015, pp. 4630-4640.

[21] N. K. Swami Naidu, Bhim Sing, "Experimental implementation of doubly fed induction generator based standalone wind energy conversion system," IEEE Trans. Ind. Appl., vol. 52, no. 4, Jul./Aug. 2016, pp. 3332-3339.

[22] G. Iwansky, W. Koczara, "Sensorless direct voltage control of the standalone slip-ring induction generator," IEEE Trans. Ind. Electron., vol. 54, no. 2, pp. 1237-1329, Apr. 2007.

[23] A. Bashar, Y. Miura, T. Ise, H. Tanaka, "Direct voltage control with slip angle estimation to extend the range of supported asymmetric loads for stand-alone DFIG," IEEE Trans. Pow. Electron., vol. 31, No.2, Feb. 2016 pp. $1015-1025$.

[24] H. Misra; A. Gundavarapu; A. K. Jain, "Control Scheme for DC Voltage Regulation of Stand-Alone DFIG-DC System," IEEE Trans. Ind. Electron., vol. 64, no. 4, pp. 2700-2708, Apr. 2017.

[25] M. F. Iacchetti, G. D. Marques, R. Perini, D. M. Sousa, "Stator Inductance Self-Tuning in an Air-gap Power Vector Based Observer for the Sensorless Control of Doubly Fed Induction Machines," IEEE Trans. Ind. Electron., vol. 61, no 1, pp. 139-148, Jan. 2014.

[26] A. Petersson, L. Harnefors, T. Thiringer, "Evaluation of current control methods for wind turbines using doubly-fed induction machines," IEEE Trans. Power Electron., vol. 20, no. 1, pp. 227-235, Jan. 2005.

[27] M. S. Carmeli, F. Castelli-Dezza, M. F. Iacchetti, Perini R., "Effect of Mismatched Parameters in MRAS Sensorless Doubly Fed Induction Machine," IEEE Trans.Power Electron., vol. 25, no. 11, Nov. 2010, pp. 2842-2851.

[28] G. D. Marques and D. M. Sousa, "Stator flux active damping methods for field-oriented doubly fed induction generator," IEEE Trans. En. Conv., vol. 27, no. 3, pp. 799-816, Sep. 2012.

[29] Liu Congwei, Weng Haiqing, Sun Xudong, Li Fahai, "Research of stability of double fed induction motor vector control system," $5^{\text {th }}$ Int. Conf. Electr. Mach. and Syst., ICEMS 2001, pp. 1203-1206 vol.2.

[30] A. Petersson, L. Harnefors, T. Thiringer, "Comparison between statorflux and grid-flux-oriented rotor current control of doubly-fed induction generators," $35^{\text {th }}$ IEEE Pow. Electr. Sp. Conf., 2004, pp. 482-486, vol.1. 